Itinéraires Itinéraires

Littérature, textes, cultures

2017-1 | 2018

Biographie et fiction

\title{
Fictionnalité et référentialité
}

Interrogations génériques : de l'autobiographie à la biofiction

Fictionality and Referentiality. Generic Speculations, from Autobiography to

Biofiction

\section{Miruna Craciunescu}

\section{OpenEdition}

\section{Journals}

Édition électronique

URL : http://journals.openedition.org/itineraires/3693

DOI : $10.4000 /$ itineraires.3693

ISSN : 2427-920X

Éditeur

Pléiade

\section{Référence électronique}

Miruna Craciunescu, «Fictionnalité et référentialité », Itinéraires [En ligne], 2017-1 | 2018, mis en ligne

le 15 février 2018, consulté le 30 avril 2019. URL : http://journals.openedition.org/itineraires/3693 ;

DOI : 10.4000/itineraires.3693

Ce document a été généré automatiquement le 30 avril 2019.

\section{(c) (i) (9)}

Itinéraires est mis à disposition selon les termes de la licence Creative Commons Attribution - Pas d'Utilisation Commerciale - Pas de Modification 4.0 International. 


\title{
Fictionnalité et référentialité
}

\author{
Interrogations génériques : de l'autobiographie à la biofiction \\ Fictionality and Referentiality. Generic Speculations, from Autobiography to \\ Biofiction
}

Miruna Craciunescu

1 Comme l'a habilement démontré Philippe Gasparini (2004 : 326-332), c'est en abordant la problématique de l'autobiographie du point de vue de sa réception, plutôt que de sa légitimité poétique ou éthique, que Philippe Lejeune s'est distingué le plus nettement de ses prédécesseurs en tant que théoricien du genre. Sous l'influence de la linguistique pragmatique, l'auteur du Pacte autobiographique (1975) n'a plus fait de la référentialité de l'écriture autobiographique une donnée qu'il conviendrait de rechercher au sein du texte lui-même, mais plutôt un effet de lecture, issu de la situation de communication que ce texte instaurait. Il est ainsi parvenu à déplacer la question difficile de l'« effet de réel » et des stratégies narratives mettant en place une illusion de référentialité, vers une interrogation portant sur l'acte de jugement qui accompagnerait le décodage d'un texte autobiographique.

2 Or, en substituant à une approche prescriptive / normative des caractéristiques du genre autobiographique une approche descriptive / typologique des situations d'énonciation qui inviteraient le lecteur à conclure qu'il se trouve face à un dispositif fictionnel ou référentiel, Lejeune a instauré un nouveau paradigme dans l'étude des genres littéraires, dont l'influence se fait nettement sentir jusque dans les travaux les plus récents d'Alexandre Gefen sur la biofiction (2004, 2014, 2015). Que l'on fonde la caractérisation générique sur des critères externes - en dégageant les principes organisateurs d'un genre à partir de ses interactions avec d'autres genres -, ou bien que l'on postule à l'inverse l'existence de critères internes de généricité, ces deux modèles tendent toutefois à négliger l'importance des échanges qui s'effectuent entre les œuvres littéraires et les réflexions génériques des commentateurs, dans ce qui s'avère être une construction commune des critères de reconnaissance de la factualité et de la fictionnalité.

3 Cet article se propose d'explorer un problème théorique lié aux considérations génériques que soulèvent l'autobiographie, l'autofiction et la biofiction dans le contexte 
plus général des débats portant sur les «frontières de la fiction » dont Françoise Lavocat a récemment fait paraître une synthèse exhaustive (2016). Je chercherai à démontrer que les différentes typologies visant à distinguer les récits de vie des trente à quarante dernières années en fonction de leur degré de fictionnalité ou de référentialité adoptent, pour la majorité, une posture que Gérard Genette (1990) a qualifiée de " conditionnaliste», liées aux études de réception, par opposition aux théories dites « essentialistes » qui, ainsi que l'expliquent René Audet et Alexandre Gefen, « fonde[nt] la fictionnalité sur une analyse immanente et taxinomique des textes ${ }^{1} »(2001$ : XIII).

Je m'intéresserai, ce faisant, à l'impact que cette approche méthodologique a produit sur la théorie des genres, en proposant que celle-ci fait aujourd'hui l'objet d'une polarisation entre deux tendances. Tandis que la première a abouti à la multiplication de catégories génériques visant à apposer un caractère normatif au " pacte de lecture » qu'instaurent des œuvres singulières, à l'instar de Fils de Serge Doubrovsky (1977) et des Vies minuscules de Pierre Michon (1984); la seconde consiste à l'inverse en l'effacement des frontières génériques au profit d'une analyse thématique de la littérature contemporaine, laquelle remplace volontiers la triade autobiographie/autofiction/biofiction par le triple « retour » du sujet, du récit et de l'histoire (Viart et Vercier 2005).

Dans un cas comme dans l'autre, j'estime que la mise en parallèle des récits autobiographiques, autofictifs et biofictifs accorde à ces derniers une cohérence qu'ils acquièrent a posteriori, moins en vertu de leurs propriétés intrinsèques qu'en raison des mutations des conditions de production et de réception des récits personnels qui ont contribué à redéfinir la relation fictionnalité / référentialité entre les décennies 1970 et 2010.

6 À l'instar d'Alexandre Gefen, il ne me semble pas pertinent de regrouper toutes les formes d'écriture de la mémoire sous le terme désormais générique d'« autofiction", pas plus qu'il n'est justifié de réduire le genre biofictif, lequel demeure avant tout « un dispositif visant à dégager de l'altérité » (Gefen 2004 : 312), à ce lieu commun de la critique qu'est rapidement devenu l'autoportrait oblique de la collection «L'Un et l'autre ». L'émergence successive de ces trois notions dans le vocabulaire critique ne m'apparaît pas directement liée à des thèmes ou à des stratégies narratives qui sous-tendraient l'ensemble de ces corpus. Aujourd'hui encore, il demeure cependant difficile d'étudier la biofiction comme un phénomène distinct de l'autobiographie et de l'autofiction en raison de l'évolution des pratiques de théorisation générique, dont certains réflexes méthodologiques ont permis à la critique de rapprocher des corpus qui diffèrent considérablement. Je crois donc qu'il importe de mettre en lumière l'évolution de ces pratiques théoriques pour comprendre d'une part les impasses auxquelles aboutissent souvent les analyses génériques soucieuses de rendre compte de l'hybridité qui caractériserait la littérature contemporaine, et pour pouvoir aborder d'autre part la biofiction autrement qu'à travers le topos critique faisant de celle-ci une « autobiographie oblique et éclatée » (Richard $1990: 87$ ).

\section{La tentation normative}

7 La théorie des genres littéraires a traditionnellement été conçue selon deux modèles. À l'approche prescriptive, à propos de laquelle Jean-Marie Schaeffer (1989:24) estime qu'elle a constitué le paradigme dominant de la critique jusqu'à la fin du xviII siècle, s'opposerait une approche descriptive, plus moderne. Tandis que la première trouve son point d'ancrage dans l'étude des conventions littéraires, la seconde se veut plus attentive 
à la singularité de chaque démarche auctoriale qu'à des critères génériques ayant valeur de règle générale.

Il est difficile d'indiquer quels ouvrages théoriques exemplifieraient au juste ces deux tendances dans la mesure où celles-ci ne s'excluent pas mutuellement, comme le souligne fort bien Schaeffer lui-même. Le nom d'Aristote est sans doute celui qui est le plus couramment associé à l'attitude prescriptive, en dépit du fait que la réception du corpus aristotélicien au XVII siècle français, relayée par les travaux d'humanistes italiens tels que Jules César Scaliger, fonctionne davantage selon un modèle normatif que ne le fait $L a$ Poétique.

Désormais, peu de critiques se réclament ouvertement de cette méthodologie, et pour cause : en établissant des normes de lecture à partir de corpus existants, les réflexions issues d'une démarche prescriptive se prêtent mal à l'identification de textes novateurs, susceptibles de modifier les pratiques d'identification générique en instaurant des points de rupture dans la tradition littéraire. Aussi, lorsqu'ils s'attachent à définir de nouvelles règles de catégorisation générique à partir de l'analyse de tels textes, les systèmes prescriptifs courent-ils le risque de s'avérer rapidement surannés.

De nombreuses tentatives pour circonscrire le phénomène autofictionnel à partir de l'analyse d'œuvres singulières témoignent de la rigidité qui semble caractériser les modèles normatifs, résistants à l'innovation générique. Ainsi, la codirectrice du site autofiction.org, Isabelle Grell, énumère-t-elle une vingtaine de néologismes ayant connu " la disgrâce et la célébrité pour des raisons obscures ", depuis les " anti-mémoires » à la Malraux jusqu'à l'« Autobiographie Génétiquement Modifiée » de Vilain, en passant par l'« auto-hétérobiographie » de Robbe-Grillet, l'« autofiction biographique » de Colonna et le « récit auto-socio-biographique » d'Annie Ernaux ${ }^{2}$.

11 Un phénomène similaire semble affecter aujourd'hui le genre biofictif, ce «fait massif d'histoire littéraire et culturelle» (Gefen 2004 : 305) qui s'est vu qualifier aussi bien de «biographie fictionnelle» (Cohn [1989] 1997) que de «fiction biographique " (Viart et Vercier 2005) et de "biographie fictive» (Dion et Fortier 2007). Cette hésitation typologique, qui n'est pas problématique en soi, le devient à partir du moment où une appellation générique n'acquiert une légitimité - et, de ce fait, une véritable utilité critique que lorsqu'elle suscite un horizon d'attente auprès d'un public assez large.

Or, précisément, une notion littéraire ne saurait être aisée à reconnaître sans avoir préalablement fait l'objet d'une conceptualisation théorique. Gasparini note à ce propos que le néologisme "autofiction" aurait mis "vingt ans à entrer dans le vocabulaire journalistique» (2008:69), ce qui correspond au délai qui s'est écoulé entre la parution initiale de la «prière d'insérer » de Fils (Doubrovsky 1977), dont la valeur de manifeste est passée pour ainsi dire inaperçue par la critique de l'époque, et celle où la Revue des libraires a affiché ce néologisme en couverture pour son numéro de l'été 1998, «signe indubitable qu'il désignait désormais un genre identifiable et largement consommable comme tel par le grand public » (Gasparini $2008: 190)$.

Ce long processus de lexicalisation est quelque peu occulté par la popularité dont jouit aujourd'hui la notion d'autofiction, ce qui explique peut-être pourquoi tant de critiques sont encore tentés par l'adoption d'un modèle normatif. Le volumineux Roman français au tournant du XXI siècle (Blanckeman et al 2004), par exemple, participe de cette tendance. Il est curieux de constater à quel point les explications visant à circonscrire le phénomène biofictionnel dans cet ouvrage collectif varient considérablement en fonction des corpus 
étudiés. Celui-ci y est compris tour à tour comme un renouvellement de l'autobiographie (Karine Gros, Béatrice Bloch); comme un glissement de l'autobiographie et de la biographie vers une forme romanesque (Irène Salas); comme une opération de résurrection des morts (Jean-François Hamel); comme un procédé herméneutique qui passe par la fiction, mais n'y trouve pas sa finalité (Dominique Viart) et comme une expérience de l'altérité pratiquant un usage spécifique de la biographie, émancipée de toute garantie référentielle (Alexandre Gefen).

En dépit de ce normativisme somme toute assez modeste, je ne crois pas cependant que des contributions de ce type puissent être perçues comme relevant d'une démarche prescriptive. À la différence des systèmes néo-aristotéliciens, les tentatives de schématisation des genres autofictionnel et biofictif ne s'articulent généralement pas au sein d'une définition étroite de la fiction, en raison de ce que Françoise Lavocat nomme «la victoire de la perspective pragmatique (John Searle et Gérard Genette) sur la proposition logico-linguistique de Käte Hamburger » (Lavocat 2016: 33) pour expliquer l'érosion des frontières entre récits fictionnels et récits factuels qui a fortement marqué le champ de la narratologie depuis le début des années $2000^{3}$.

Celle-ci a donné lieu à ce que des chercheurs comme Jan Alber et Monika Fludernik (2010) appellent désormais la «narratologie postclassique ", d'après la définition qu'en a donnée David Herman dans son introduction à l'important volume Narratologies :

La narratologie postclassique (qui ne devrait pas être confondue avec les théories poststructuralistes de la narration) contient la narratologie classique [issue du structuralisme français] comme un de ses «moments » tout en étant marquée par une profusion de nouvelles méthodologies et d'hypothèses de recherche, lesquelles ont donné lieu à de nouvelles perspectives portant sur les formes et les fonctions de la narration elle-même ${ }^{4}$. (Herman 1999 : 2-3)

\section{Narratologie " postclassique » et théorie des genres: le cas de l'autobiographie}

16 Caractérisées par un " tournant narratif » (narrative turn), que l'on appelle parfois aussi le phénomène du storytelling, les analyses issues de la narratologie postclassique débordent largement du domaine des études littéraires pour englober l'ensemble des textes factuels et fictionnels qui auraient pour caractéristique commune de partager une même identité narrative, depuis la présentation des événements du 11 septembre dans les médias (Hansen 2005: 285-287) jusqu'à la transmission d'informations dans les récits historiographiques, les histoires de cas médicales, les transcriptions de procès et la publicité. De ce fait, ces travaux effectuent couramment une adéquation entre narrativité et fictionnalité en postulant, à la suite de Hayden White (1973, 1987, 1999), que toute «mise en récit» de faits ou d'événements engendre nécessairement un processus de fictionnalisation, ce qui a notamment amené Monika Fludernik à concevoir la création de récits fictionnels comme un acte «naturel », comparable à une conversation spontanée (1996: 13-14). Aussi Luc Herman et Bart Vervaeck considèrent-ils, dans le même ordre d'idées, que « tandis que $1[$ a narratologie classique] structuralis[te] a tenté d'articuler une théorie générale de la narrativité, la narratologie postclassique préfère étudier les circonstances faisant de chaque acte de lecture un acte différent » $\left(2005: 450^{5}\right)$; ce qui, il convient de le noter, rend l'adoption d'une démarche descriptive pour ainsi dire inévitable. 

théorie des genres et les nouvelles perspectives en narratologie pendant les décennies 1970 à 2010, et surtout dans quelle mesure leurs mutations respectives résultent d'une influence réciproque, ou tout simplement d'une évolution parallèle. Bien avant que David Herman ne donne le coup d'envoi de la narratologie postclassique, le paradigme conversationnel avait déjà amené certains théoriciens des genres à concevoir le texte littéraire comme un support communicationnel pourvu d'une intentionnalité ${ }^{6}$, au sein duquel la question de l'identité générique serait indissociablement liée au contexte accompagnant l'acte discursif qu'instaure un texte littéraire, ce dont les travaux de Lejeune témoignent de manière exemplaire.

, Martin Löshnigg (2010: 256) note que ce n'est guère en raison d'un soupçon poststructuraliste généralisé envers les notions de «vérité " et d' "authenticité » que la distinction entre fait et fiction n'attire plus autant l'attention des théoriciens du genre autobiographique, mais bien parce que la narrativité est désormais perçue comme un facteur décisif dans la construction de l'identité auctoriale. Cette conclusion, directement héritière de la réflexion lejeunienne, en inverse pourtant les prémisses. En effet, loin d'affirmer qu' « une identité est ou n'est pas » (Lejeune 1975: 15) et que tout doute concernant l'adéquation auteur / narrateur / personnage amènerait le lecteur à conclure qu'il se trouve face à un pacte fictionnel, et donc non autobiographique, la narratologie postclassique considérerait à présent l'autobiographie comme un acte fondateur de l'identité auctoriale (Löshnigg $2010: 259$ ).

Ce faisant, elle semble avoir poursuivi la voie empruntée par Lejeune lorsque celui-ci s'est adonné à une réévaluation critique de ses premiers travaux en remarquant que «l'indéniable aspect normatif du "Pacte" [de 1975 tenait] essentiellement à la présentation tranchée du problème de l'identité » (Lejeune $1986: 20$ ), alors même que sa notion d'"espace autobiographique" avait donné lieu à une multitude de positions intermédiaires qui tolèrent, pour ne pas dire favorisent, l'existence d'ambiguïtés dans l'identification générique. En d'autres termes, dans le débat essentiellement narratologique portant sur les "frontières de la fiction", Lejeune a explicitement assumé, depuis le " Pacte autobiographique bis », ce que Marie-Laure Ryan a par la suite appelé un modèle " analogique », lequel conceptualise la distinction entre fictionnalité et référentialité en termes de degrés, par opposition à un modèle binaire (ou " digital $\left.{ }^{7} »\right)$ qui l'envisage comme une frontière nettement délimitée :

Le modèle digital considère les modes fictionnel et non fictionnel de la lecture comme les deux voies d'un aiguillage ferroviaire: le lecteur peut soumettre un texte donné à chacun des deux modes. Le sous-titre générique nous aiguille dans une voie ou dans une autre, mais il est toujours possible d'ignorer l'intention de l'auteur ou de sauter d'une voie à l'autre. [...] Un tel changement de voie ne peut être expliqué dans un modèle analogique, puisque l'idée d'un axe linéaire continu présuppose soit un seul mode de lecture susceptible de degrés, soit deux modes qui croissent et décroissent aux dépens de l'autre. (Ryan 2001 : 33)

Il existe actuellement plusieurs terminologies concurrentes pour caractériser ces deux postures, dont la plupart ne se recoupent pas tout à fait, en raison des présupposés théoriques adoptés par chaque théoricien. Françoise Lavocat par exemple s'est montrée critique envers la nomenclature connotée de Thomas Pavel (1988 [1986]), qui a distingué les théories postulant une différence entre fait et fiction de celles qui admettent leur chevauchement ou leur brouillage, en qualifiant les premières de "ségrégationnistes » et les secondes, d'«intégrationnistes ». Dénonçant ce qui lui a semblé être une absence de 
neutralité à l'égard du binarisme, au sein d'un ouvrage qui s'avère être un plaidoyer en sa faveur, Lavocat (2016: 34) a proposé de rebaptiser ces deux notions «monisme » et «différentialisme »- en précisant toutefois qu'elles sont plus larges que celles de Pavel, dans la mesure où elle estime que la théorie pavélienne de la fiction «tend vers le monisme, mais repose sur une différenciation de degré, et non de nature, entre factuel et fictionnel, réalité et imaginaire » (ibid. : 385).

On observe ici un phénomène similaire à la multiplication de néologismes qui a caractérisé la conceptualisation du genre autofictif, pour des raisons qui m'apparaissent semblables. Dans un cas comme dans l'autre, la consécration d'une terminologie plutôt qu'une autre implique l'adoption d'une posture particulière à l'égard des pratiques de reconnaissance de la fictionnalité et de la référentialité, qu'il s'agisse uniquement d'un contexte d'analyse textuelle ou bien, de manière plus globale, de la distinction entre fait et fiction, " vérité » et « fabrication ", « invention » ou « mensonge ». Or, cette distinction est aussi bien historique que culturelle, ce que révèlent éloquemment les analyses de Dominique Viart (2002) et de Daniel Madelénat (2007) en confrontant les pratiques française (ou « continentale ») et anglo-saxonne de la biographie.

\section{Les frontières de la fiction : le cas de la biographie}

Dans l'article liminaire d'un numéro spécial consacré aux « Paradoxes du biographique » dans la Revue des sciences humaines, Dominique Viart (2002) a tout d'abord associé la fascination contemporaine envers le minuscule dans les récits de vie à ce qu'il a appelé les "gestions du détail» que les pratiques française et anglo-saxonne de la biographie mettraient chacune traditionnellement en œuvre. Daniel Madelénat (2007) a par la suite repris cette distinction en l'identifiant comme deux "régimes de vérité » permettant d'accentuer la référentialité des textes biographiques selon des conventions culturelles qui varient en fonction des attentes de chaque public.

Ainsi, tandis que la tradition anglo-saxonne tendrait à produire des biographies «monumentales [...] qui visent à l'exhaustivité, traquent l'infime pour l'accumuler et produisent finalement une restitution de vie comme quantité » (Viart 2002: 15), la tradition française se caractériserait en revanche par son goût marqué pour l'herméneutique préférant, à l'accumulation de faits insignifiants, subordonner un nombre restreint de «biographèmes » à l'interprétation d'un projet existentiel. Selon Madelénat, la mauvaise réception dont la biographie de Georges Perec par David Bellos a fait l'objet en France en 2003 s'expliquerait par cette différence culturelle, attribuant à la quête de vérité qui sous-tend tout projet biographique des procédés d'écriture radicalement différents.

Comme l'avait précédemment souligné Dominique Viart, ces procédés d'écriture se distingueraient le mieux par leur usage du détail : au «factuel paratactique » anglo-saxon prônant l'anecdote et la dénotation réaliste, s'opposerait la « fascination [continentale] pour les continuités profondes d'une vie » visant une vérité qui est « dévoilement » ainsi qu'une connaissance qui est «symbolique " (Madelénat 2007 : 298). Une telle dichotomie se retrouve aujourd'hui dans l'écriture biofictive dont Marcel Schwob aurait articulé les prémisses dans sa préface désormais célèbre aux Vies imaginaires (1896), où les styles « anglo-saxon » de James Boswell et «continental » de John Aubrey servent à la fois de modèle et de contre-modèle à la création d'un «nouvel imaginaire biographique ». À en 
croire les contributions réunies dans l'ouvrage d'Anne-Marie Monluçon et Agathe Salha (2007), ce nouvel imaginaire biographique aurait trouvé son œuvre phare dans les Vies minuscules de Pierre Michon (1984) en retenant d'Aubrey la forme courte, et de Boswell la singularité des anecdotes, permettant de « raconter avec le même souci les existences uniques des hommes, qu'ils aient été divins, médiocres ou criminels » (Schwob $1896: 21$ ).

Cette opposition entre les "régimes de vérité » français, ou continental, et anglo-saxon, me paraît être un bon exemple de l'aisance avec laquelle des procédés narratifs d'identification de la fictionnalité et de la référentialité se transfèrent d'un plan textuel, essentialiste - qui est celui de la narratologie classique, puisque celle-ci tend à étudier le fonctionnement de procédés narratifs indépendamment des contextes culturels qui informent leurs conditions de production et de réception - à un plan extratextuel, conditionnaliste, associé, pour sa part, à la narratologie "postclassique». Les recherches issues de ce courant tendent en effet à démontrer qu'il n'y aurait pas de technique narrative propre aux récits référentiels, puisque l'identification de la nature fictionnelle ou référentielle d'un énoncé donné résulterait entièrement d'un processus externe, intervenant au moment de la réception de cet énoncé. Cela suppose que la différence entre ce qui est fictionnel et ce qui est référentiel ne dépendrait donc pas du lien qu'un énoncé donné entretient avec le monde référentiel auquel il se rapporte, mais bien des conventions culturelles qui déterminent quels procédés narratifs sont recevables, et quelles techniques discursives il convient à l'inverse de proscrire au sein de pratiques discursives référentielles, telles que l'historiographie.

D'une part, le «factuel paratactique ${ }^{8}$ » anglo-saxon, par exemple, dénote la référentialité en créant l'illusion qu'il s'agit essentiellement d'une compilation de données, de références et de documents d'archives, lesquelles n'auraient que minimalement fait l'objet d'une réorganisation, et donc d'une "mise en récit ", de la part du biographe. Les présupposés théoriques qui sous-tendent cette tradition biographique sont donc en grande partie celle de la narratologie postclassique, dans la mesure où la narrativité y devient synonyme de fictionnalité, et que l'accumulation - qui est en soi un procédé narratif, ce qu'on oublie parfois - vise à diminuer la part de subjectivité intervenant dans l'écriture biographique. Le cadre de référence définissant la "vérité » est donc implicitement celui du protocole de travail en laboratoire qui caractérise les expériences scientifiques: celles-ci ne sont considérées objectives que parce qu'elles sont reproduisibles, ce qui implique que toute personne qui tenterait de réécrire une biographie anglo-saxonne rédigée selon un modèle factuel paratactique procéderait à une accumulation des mêmes données, produisant au bout du compte un document similaire.

D'autre part, en se basant sur un modèle analytique subordonnant les faits narrés à une hypothèse englobante, la biographie continentale adopte implicitement pour cadre de référence de la "vérité » une définition pour tout dire romantique du travail de l'artiste, dont la connaissance du monde surviendrait à la suite d'un dévoilement, ou aletheia ( Madelénat 2007 : 298), qui fonde sa légitimité épistémologique dans la subjectivité. Le but du biographe continental n'est donc pas de produire un récit reproduisible, mais au contraire unique dans le lien de sympathie qu'il crée entre un biographe et son biographé. Pour parvenir à faire « sortir » ce dernier de l'oubli, le biographe a pour devoir de comprendre celui dont il met en récit l'existence, afin d'en sélectionner les événements de sa vie qu'il considère avoir été les plus marquants. Il doit ainsi chercher à modeler sa propre voix narrative sur celle de son biographé afin d'adopter une perspective, sinon homodiégétique, du moins aussi près que possible de celle qu'aurait 
adoptée son biographé au moyen d'un mimétisme formel, ou bien en adoptant des sensibilités (esthétiques, philosophiques, idéologiques) analogues. Le résultat visé n'est pas une accumulation de faits, mais leur simulation, conformément à la définition de la biographie proposée par Paul Murray Kendall, qui serait une «simulation, par des mots, d'une vie d'homme, à partir de tout ce qu'on connaît sur cet homme » $(1965: 15)$. Or, ce faisant, une biographie "vraie", au sens où l'entend la tradition continentale, mettrait en œuvre ce que Dorrit Cohn ([1989] 1997) estime être le principal marqueur « interne » de fictionnalité au sein d'un texte, c'est-à-dire l'application systématique de techniques de focalisation interne à des personnages réels, laquelle a pour effet de confondre les propos du biographe avec ceux de son modèle historique, au point où le lecteur n'est, parfois, plus à même de dire « où s'arrête le fait établi, et où commence l'invention » (ibid. : 34).

De là résulte qu'un même procédé narratif peut constituer un marqueur de fictionnalité ou de référentialité, dépendamment du contexte culturel, et surtout théorique, qui informe sa réception auprès de la critique.

\section{Problèmes terminologiques, du modèle binaire au modèle " analogique »}

Quelle terminologie permettrait de cerner le plus efficacement les enjeux qui sont à l'œuvre dans ces différentes manières de penser les « frontières de la fiction " ? J'ai déjà mentionné que l'opposition entre «essentialisme » et "conditionnalisme» (Audet et Gefen 2001), "ségrégationnisme» et «intégrationnisme " (Pavel [1986] 1988), et «monisme » et « dualisme » (Lavocat 2016) ne se recoupe pas tout à fait, tout comme on ne saurait réduire ces différentes notions aux deux modèles d'appréhension de la fiction proposés par Marie-Laure Ryan (2001). En vérité, si ces terminologies ne permettent pas de décrire les mêmes phénomènes, c'est parce qu'elles abordent la fictionnalité selon des perspectives distinctes. Tandis que Françoise Lavocat interroge, dans Fait et fiction (2016), le problème qui consiste à distinguer ces deux notions selon une perspective ontologique, les contributions réunies par René Audet et Alexandre Gefen dans Frontières de la fiction (2001) - lequel s'ouvre d'ailleurs sur un article de Thomas Pavel - explorent pour la plupart la problématique éponyme selon une perspective logique. Or, celle-ci vise à différencier non pas la nature du réel de la nature de l'imaginaire, mais bien les propositions référentielles des propositions fictionnelles, selon un schéma hérité de la linguistique pragmatique qui semble informer autant les réflexions génériques contemporaines que les analyses issues de la narratologie dite " postclassique ».

Il est toutefois difficile de séparer tout à fait ces deux perspectives, car la définition pragmatique de la fiction comme une " feintise ludique partagée » (Schaeffer 1999) n'est pas entièrement dépourvue de conséquences ontologiques. C'est ce que souligne fort bien Pavel en indiquant qu'une conception de l'univers fictionnel qui réduit celui-ci à un acte de langage, dont la valeur performative se limiterait à un jeu gouverné par «une convention culturelle stable» (2001:5), risque de nous faire perdre de vue une de ses finalités les plus importantes, qui est de "nous exhorter à réfléchir à l'organisation normative et axiologique du monde que nous habitons » (ibid. : 10). Le choix de concevoir le langage comme un système autonome aboutissant à la création d'univers autopoiétiques n'est pas neutre : au-delà des difficultés théoriques que soulève une telle 
posture sur un plan narratologique lorsque l'on cherche à définir des critères distinctifs de référentialité, les théories monistes posent un véritable problème sur le plan éthique, puisqu'elles ne permettent pas d'établir de distinction, par exemple, entre un document authentique et un document qui résulte d'une forgerie. Carlo Ginzburg souligne qu'un tel problème « est de nature à mettre les sceptiques mal à l'aise puisqu'il implique [...] cette réalité externe que les guillemets n'arrivent pas à exorciser» $(2010: 16)$; aussi de nombreux historiens se sont-ils opposés à l'adéquation entre fictionnalité et narrativité.

Le «tournant linguistique» (linguistic turn), dont Hayden White constitue l'un des principaux défenseurs, a effectivement fait l'objet de nombreuses critiques en raison du relativisme ontologique auquel conduirait la volonté de mettre entre parenthèses la valeur de vérité et les conséquences pratiques immédiates d'un discours, afin de se concentrer exclusivement sur son mode de fonctionnement. Dans un ouvrage collectif qui explore la réception des thèses de White dans les études historiques portant sur l'Holocauste, Carlo Ginzburg remarque à ce propos que, si la vérité d'un discours historique consistait en son efficacité, l'interprétation sioniste de l'Holocauste ne se distinguerait de celle de Faurisson qu'en raison de sa capacité à justifier un large éventail de politiques israéliennes, là où l'interprétation de Faurisson ne permet pas aux Palestiniens d'articuler une contre-idéologie capable de s'opposer efficacement à la mise

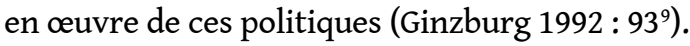

Quoi qu'il en soit, le choix d'adopter une perspective logique et linguistique face à la question « qu'est-ce que la fiction?» semble entraîner l'adhésion plus ou moins explicite, sur un plan ontologique, à une perspective moniste qui n'accorde pas à la fictionnalité et à la référentialité des identités distinctes. Sur le plan de l'analyse textuelle, cela se traduit par la mise en œuvre d'une démarche conditionnaliste qui suppose que la fiction résulterait d'un usage que l'on choisit de faire du texte, et non de ses propriétés intrinsèques. En postulant d'une part l'ubiquité de la narrativité et en effectuant, d'autre part, une adéquation entre narrativité et fictionnalité, les analyses issues de la narratologie postclassique s'opposent de ce fait à ce que Lavocat appelle le "différentialisme", dans la mesure où elles étendent la définition de la fiction à l'ensemble des productions discursives et non discursives comportant une part de narrativité.

Leurs critères d'identification de la fictionnalité tendent ainsi à s'effectuer selon un modèle "intégrationniste " plutôt que "ségrégationniste » d'après la terminologie de Pavel, ou « analogique » plutôt que « digital » ou binaire, d'après celle de Ryan. L'accord de « feintise ludique partagée » qui s'établit par exemple à la lecture d'un texte fictionnel ne peut effectivement pas être conceptualisé selon une opposition binaire entre « fait » et «fiction »: la conception lejeunienne d'«espace autobiographique » témoigne à cet égard, contre la binarité tant critiquée du "pacte», du fait que la référentialité est fréquemment conçue en termes de degrés dans la théorie des genres littéraires, ce qui constitue toute la difficulté d'établir des critères normatifs permettant de distinguer l'autobiographie de l'autofiction.

Dans le même ordre d'idées, ce n'est pas parce que la biographie de Georges Perec par David Bellos ne correspondait pas au «régime de vérité » continental qu'elle a, pour autant, fait l'objet d'une réception fictionnelle. Cependant, l'analyse de Madelénat (2007) révèle que, même si l'on admet que l'identification référentielle de ce texte s'est effectuée par le biais de facteurs extratextuels, tels que le paratexte et la réception médiatique de l'œuvre, c'est avant tout parce que la biographie de Bellos ne mettait pas en œuvre les 
procédés narratifs qui sont traditionnellement associés à la référentialité biographique française qu'elle a fait, en France, l'objet de critiques acerbes.

Tout porte donc à croire que l'opposition entre monisme et différentialisme, conditionnalisme et essentialisme, ou intégrationnisme et ségrégationnisme n'informe pas les réflexions génériques de façon binaire, puisque la reconnaissance « conditionnaliste» de la nature historique et culturelle de la fictionnalité n'exclut pas l'identification «essentialiste» de procédés narratifs à travers lesquels s'exprime cette référentialité.

\section{La tentation descriptive}

Cela dit, il me semble évident que, dans le paradigme qui informe actuellement la théorie des genres littéraires depuis les travaux de Lejeune, la démarche essentialiste ne jouit pas d'une popularité égale à celle du conditionnalisme. Ainsi, l'ascendant exercée par les travaux de Searle sur la théorie des genres littéraires des quarante dernières années a régulièrement incité la critique à accepter le postulat voulant qu'«il n'y [ait] pas de propriété textuelle, syntaxique ou sémantique qui permette d'identifier un texte comme une œuvre de fiction» (Searle [1975] 1982: 109); ce qui, au dire de Lavocat, aurait " supplanté » les théories de Käte Hamburger ([1957] 1986) sans que l'on ait démontré leur caractère inopératoire (Lavocat $2016: 34$ ).

Il faut dire que les narratologues qui se réclament de cette théoricienne allemande sont en effet peu nombreux ${ }^{10}$. Dorrit Cohn compte parmi les plus connus, en dépit du caractère volontairement restrictif de sa perspective qui «refuse de considérer que la fiction pourrait être autre chose que du roman " (ibid. : 18). Contrairement aux travaux issus de la narratologie postclassique, cela l'a amenée à concevoir la distinction entre fictionnalité et référentialité de manière binaire, en définissant la fiction comme "un récit non référentiel [excluant] donc toutes les propositions générales vérifonctionnelles qui caractérisent le discours théorique, philosophique, explicatif, spéculatif ou critique " (Cohn [1999] $2001: 28$ ).

39 Il est aisé de concevoir pourquoi l'adoption d'une définition étroite de la fiction favorise les réflexions génériques prescriptives au détriment des démarches descriptives. En ce qui a trait à la théorie des genres littéraires, la marginalisation de la perspective de Hamburger contre celle de Searle n'a pas seulement encouragé la critique à favoriser les facteurs externes de fictionnalité, puisque l'identification générique a elle-même subi un processus d'externalisation, dans la mesure où les traits caractéristiques d'un genre littéraire ne sont, bien souvent, plus à rechercher au sein des textes eux-mêmes, mais varient plutôt en fonction de leur appareil paratextuel ou de leur réception critique et médiatique. C'est ce qu'illustre Schaeffer en définissant la constitution d'une classe textuelle comme « un processus discontinu lié à une causalité externe » $(1989: 71)$, ce qui la distinguerait nettement de la classification biologique qui lui a longtemps servi de modèle :

De ce fait, il est vain d'espérer pouvoir déduire causalement les classes génériques à partir d'un principe interne sous-jacent: même s'il existe une compétence générique, elle ne saurait être que celle des auteurs et des lecteurs, et non pas celle des textes. (Ibid. : 74) des caractéristiques génériques de la biofiction en favorisant les explications post factum. 
Celles-ci consisteraient, par exemple, à conclure que, si l'autofiction était peut-être « une issue aléatoire à l'impossibilité de l'autobiographie et à l'illusion d'y trouver une identité narrative forte»-comme l'a fait Jacques Lecarme (2004: 19) en définissant ainsi la position de Régine Robin (1998) -, la biofiction pourrait n'être à son tour qu'une variante de l'écriture autofictionnelle, laquelle aurait alors pour particularité d'exposer «le lien intime et fort » qui se crée entre un auteur et "son héros secret ", pour reprendre les termes du texte qui se trouve sur le rabat de la couverture de tous les livres parus dans la collection «L'Un et l'autre ", dont Agnès Blesch et Pauline Mulin ont commenté la valeur de manifeste ${ }^{11}$.

41 En dépit de la grande diversité des œuvres qui ont retenu l'attention de la critique à titre d'autobiographies, d'autofictions ou de biofictions, l'ensemble de cette production littéraire peut ainsi être placée dans un même continuum plutôt que de faire l'objet d'une tripartition laissant entendre que ces corpus appartiendraient à des phénomènes distincts les uns des autres. Une telle interprétation offre l'avantage de dégager des lignes directrices fortes aussi bien dans la théorie des genres littéraires que dans la production littéraire française depuis le «tournant » des années 1980, en s'organisant l'une comme l'autre autour de quelques axes de réflexion thématiques liés au triple retour du sujet, du récit et de l'histoire, qui aurait marqué la fin des avant-gardes. C'est ce que font plusieurs études qui s'appliquent à "donner des repères dans ce qui fonde la littérature aujourd'hui », dont La Littérature française au présent de Dominique Viart et de Bruno Vercier (2005) est sans doute la plus emblématique.

Or, cette quête des causes profondes des mutations que l'on observe dans les pratiques d'écriture contemporaines à partir des années 1980 s'est avérée tout aussi défavorable à la théorie des genres littéraires que ne l'est, sur le plan théorique, la double externalisation des critères d'identification générique ainsi que des critères de fictionnalité.

Il est en effet beaucoup plus difficile d'établir des critères permettant de distinguer in abstracto des textes appartenant à un régime factuel (autobiographie, biographie) des textes appartenant à un régime fictionnel (autofiction, biofiction) en fonction d'une situation d'énonciation impliquant un lecteur, un texte et un auteur, plutôt qu'à partir d'une seule analyse de texte. C'est bien ce qui explique la perméabilité des critères d'identification générique: pour parvenir à décrire l'ensemble des pactes de lecture possibles tout en tenant compte de la singularité de chacun, il est nécessaire de multiplier les propositions typologiques afin d'établir des normes susceptibles d'informer à la fois la lecture de corpus existants ainsi que la composition d'œuvres à venir.

Les démarches prescriptives / normatives s'avèrent intenables dans un tel contexte puisque, en l'absence de marqueurs invariables de fictionnalité, il y a pour ainsi dire autant de degrés de fictionnalisation de récits référentiels qu'il n'y a de textes biographiques, autobiographiques, autofictifs et biofictifs. Aussi, l'adoption du postulat searlien conduit-il, sur le plan méthodologique, à une démarche descriptive / typologique qui tente de dégager les principes organisateurs d'un genre à partir de ses interactions avec d'autres genres.

45 Il me semble pour finir que la multiplication de néologismes et de typologies qui caractérise la théorie des genres littéraires depuis Lejeune résulte d'un compromis entre la posture moniste niant la possibilité de distinguer entre fait et fiction, implicite chez Searle, et la posture différentialiste postulant l'existence de «marqueurs» de 
fictionnalité, défendue par Dorrit Cohn (1997, [1999] 2001) dans la lignée de Hamburger. L'adoption d'une posture moniste peut difficilement aboutir à autre chose qu'à une dissolution des frontières génériques au profit d'une hybridation généralisée, ce qu'explique éloquemment Dominique Viart, pour qui le brouillage conceptuel résultant d'une conception analogique de la fictionnalité aurait "créolisé » l'écriture contemporaine au point de remettre définitivement en question la pertinence de la critique générique :

Toute entreprise [qui vise à considérer les genres littéraires] se perd en catégories, étiquettes et classements, dont les marges se brouillent. S'il faut inventer un nouveau nom pour chaque nouvelle œuvre, que devient le classement? Nous savons tous que l'emploi de la première personne «ne garantit pas le sujet de l'énonciation » [...] et que c'est toujours en quelque façon de soi-même aussi que l'on parle, quelle que soit la forme du discours. [...] Sans doute faudrait-il alors substituer à la critique des genres, au moins pour la littérature contemporaine, une critique des enjeux - et des moyens mis en œuvre pour les atteindre. (Viart 2002: 28-29)

Un tel compromis viserait donc à soustraire la théorie des genres de l'impasse à laquelle la condamne l'adoption de critères externes de fictionnalité, en la contraignant à systématiser des expériences de lecture qui ne sauraient être que singulières.

\section{Pourquoi opter, malgré tout, pour une approche générique?}

47 Compte tenu de ces considérations, j'estime que les liens encore mal connus qu'entretiennent l'autobiographie, l'autofiction et la biofiction avec les débats narratologiques et historiographiques portant sur les frontières de la fiction justifient sans doute davantage leur rapprochement sur un plan théorique que sur un plan littéraire. C'est ce dont témoigne notamment le fait que l'étude des textes qui ont été rapprochés par la critique à titre de "biofictions", plutôt que de se limiter à quelques traits aisément repérables, a généré une constellation d'ouvrages multipliant les traits spécifiques à chaque démarche auctoriale ${ }^{12}$.

Cela dit, il me semble que l'analyse tripartite demeure malgré tout utile en tant que paradigme permettant d'appréhender l'évolution de la littérature contemporaine, dans la mesure où une étude comparée de la consécration des genres autofictif et biofictif met en lumière de nombreux points communs dans la manière dont ils ont été institués en tant qu'objets littéraires. Il suffit de rappeler quelques-unes des étapes du long processus de lexicalisation du terme "autofiction " pour constater qu'un tel rapprochement peut s'avérer utile à la compréhension du phénomène biofictif.

Après que l'autofiction a pris quelque temps un sens « étroit », ce genre s'est vu attribuer une acception «maximaliste » à la suite des débats qui ont opposé Serge Doubrovsky à Vincent Colonna au sujet de la nature fictionnelle des événements narrés ${ }^{13}$. Ainsi, ce n'est que lorsque l'autofiction a été comprise comme une forme d'autofabulation que l'«amnésie initiale» de la critique - laquelle avait tout d'abord identifié dans le néologisme proposé par Doubrovksy un phénomène inédit et pour tout dire marginal, de la production littéraire contemporaine -, a cédé la place à une démarche qui se proposait de situer l'émergence du genre autofictif dans la longue durée. 

définition du genre de l'autofiction de façon à englober l'ensemble des productions textuelles au « contenu narratif [...] authentiquement fictionnel » (Genette 1991 : 86-87) qui formuleraient ce que Gérard Genette estimait être un pacte de lecture « délibérément contradictoire ${ }^{14}$ ", en raison de l'hybridation des marqueurs de référentialité et de fictionnalité par lesquels l'auteur d'une œuvre au contenu « manifestement imaginaire » effectuerait néanmoins l'association auteur-narrateur-protagoniste, identifiée par Lejeune comme étant le propre du régime autobiographique.

51 Or, alors même que l'on assiste, dans les années 1990, au foisonnement d'une terminologie visant à circonscrire le phénomène "éminemment contemporain " du retour du sujet dont l'autofiction serait révélatrice, la propension à voir dans ce genre un phénomène d'époque, emblématique du Zeitgeist postmoderne, coexiste avec l'impulsion contraire qui consiste à en faire l'aboutissement d'une tradition littéraire dont les origines se sont vues déplacées jusqu'au XIV ${ }^{\mathrm{e}}$ siècle - ce qui implique que l'autofiction, loin de relever uniquement d'un phénomène récent, informerait depuis déjà longtemps les processus largement culturels de distinction entre un texte rédigé en régime factuel et un texte rédigé en régime fictionnel.

52 La prise en charge théorique de la notion de "biofiction » témoigne d'une évolution similaire. Si la constitution d'un corpus biofictif relève, il est vrai, à la fois d'un phénomène littéraire dont témoignerait la parenté de certaines œuvres, à l'instar de la «triade» formée par Marcel Schwob, Pierre Michon et Gérard Macé, ainsi que d'un phénomène éditorial qui tend à soumettre cette même production aux contraintes d'une poignée de collections, placées sous l'égide de quelques grandes maisons éditoriales françaises («L'un et l'autre » chez Gallimard, « Une journée particulière » chez JC Lattès, «Elle était une fois » chez Laffont, « Traits et portraits » chez Mercure de France, etc.), il n'en demeure pas moins que l'hétérogénéité de ce corpus, et les difficultés auxquelles l'on se heurterait conséquemment en cherchant à en définir les limites, s'explique à mon avis par le fait que la création d'un horizon d'attente biofictif procède avant tout d'un phénomène critique.

53 Étant donné la marginalisation actuelle de l'analyse générique au profit d'une analyse thématique de la littérature contemporaine, il est aisé de comprendre pourquoi les biofictions ont fini par prendre une acception particulière à chaque étude qui leur a été consacrée : les variations de leur corpus dépendent en réalité des intérêts de recherche de ses commentateurs. La biofiction a ainsi été liée à l'histoire de la biographie et à ses récents développements à l'ère du «tournant narratif » (Madelénat); à la résurgence de la figure de l'auteur dans la littérature contemporaine (Buisine, Dion, Fortier), ou à la « relation biographique » qui a participé à la littéralisation croissante de la biographie en faisant de ce genre factuel un artefact verbal (Bruce Nadel, Boyer-Weinmann); à la fonction heuristique des "fictions critiques" (Viart), ainsi qu'à la manière dont ces dernières, en imitant un discours factuel, permettraient ou non l'identification de marqueurs internes de fictionnalité (Cohn, Lavocat).

Il s'agit incontestablement d'un champ d'étude en pleine expansion. Sans doute connaîtra-t-il d'autres mutations importantes dans les années à venir. 


\section{BIBLIOGRAPHIE}

Alber, Jan et Fludernik, Monika, 2010, Postclassical Narratology: Approaches and Analyses, Columbus, Ohio State University Press.

Audet, René et Gefen, Alexandre, 2001, "Présentation », dans R. Audet et A. Gefen (dir.), Frontières de la fiction, Québec, Nota Bene, Bordeaux, Presses Universitaires de Bordeaux, p. VII-XVII.

Banfield, Ann, [1982] 1995, Phrases sans parole. Théorie du récit et du style indirect libre, trad. de l'anglais par Cyril Veken, Paris, Seuil.

Blanckeman, Bruno, Mura-Brunel, Aline et Dambre, Marc (dir.), 2004, Le Roman français au tournant du XXI ${ }^{e}$ siècle, Paris, Presses Sorbonne Nouvelle.

Boyer-Weinmann, Martine, 2005, La Relation biographique, Seyssel, Champ Vallon, coll. « Enjeux contemporains ».

Bruce-Nadel, Ira, 1984, Biography: Fiction, Fact and Form, New York, St. Martin's Press.

Buisine, Alain, 1991, « Biofictions », Revue des sciences humaines, $\mathrm{n}^{\circ} 224$, « Le Biographique », p. 7-13.

Cohn, Dorrit, [1989] 1997, « Vies fictionnelles, vies historiques : limites et cas limites », trad. fr. J.-

P. Mathy, Littérature, $\mathrm{n}^{\circ} 105$, p. 24-48.

Cohn, Dorrit, [1999] 2001, Le Propre de la fiction, trad. fr. C. Hary-Schaeffer, Paris, Seuil, coll. « Poétique ».

Colonna, Vincent, 1989, L'Autofiction : essai sur la fictionnalisation de soi en littérature, thèse sous la direction de Gérard Genette, Paris, École des Hautes études en sciences sociales.

Dion, Robert, Fortier, Frances, Havercrot, Barbara, LüseBrink, Hans-Jürgen (dir.), Vies en récit. Formes littéraires et médiatiques de la biographie et de l'autobiographie, Québec, Nota Bene, coll. « Convergences ».

Doubrovsky, Serge, 1977, Fils, Paris, Galilée.

Fludernik, Monica, 1996, Towards a "Natural" Narratology, Londres, Routledge.

Gasparini, Philippe, 2004, Est-il je ? Roman autobiographique et autofiction, Paris, Seuil.

Gasparini, Philippe, 2008, Autofiction. Une aventure du langage, Paris, Seuil.

Gefen, Alexandre, 2004, « Le genre des noms. La biofiction dans la littérature française contemporaine », dans B. Blanckeman, A. Mura-Brunel et M. Dambre (dir.), Le Roman français au tournant $d u \mathrm{XXI}^{e}$ siècle, Paris, Presses Sorbonne Nouvelle, p. 305-319.

Gefen, Alexandre, 2014, Vies imaginaires : de Plutarque à Michon, Paris, Gallimard, coll. « Folio classique ».

Gefen, Alexandre, 2015, Inventer une vie : la fabrique littéraire de l'individu, préface de Pierre Michon, Paris, Les Impressions Nouvelles.

Gefen, Alexandre et Audet, René (dir)., 2001, Frontières de la fiction, Québec, Nota Bene. Genette, Gérard, 1990, «Fictional Narrative, Factual Narrative », Poetics today, vol. XI, $\mathrm{n}^{\circ} 4$, « Narratology Revisited II », p. 755-774. 
Genette, Gérard, 1991, Fiction et diction, Paris, Seuil.

Ginzburg, Carlo, 1992, « Just One Witness », dans S. Friedlander (dir.), Probing the Limits of Representation: Nazism and the "Final Solution", Cambridge, Ma, Harvard University Press, p. 82-96.

Ginzburg, Carlo, 2010, Le Fil et les Traces : vrai faux fictif, trad. Martin Rueff, Paris, Verdier.

Hamburger, Käte, [1957] 1986, Logiques des genres littéraires, trad. de l'allemand par Pierre Cadiot, Paris, Seuil.

Hansen, Per Krogh, 2005, « When Fact Becomes Fiction: Facts, Fiction and Unreliable Narration », in Skalin (dir.), Fact and Fiction in Narrative: An Interdisciplinary Approach, Örebro, O. University Press, p. 285-307.

Herman, David, 1999, « Introduction. Narratologies », dans D. Herman (dir.) Narratologies. New Perspectives in Narrative Analysis, Columbus, Ohio State University Press, p. 1-30.

Herman, Luc et Vervaeck, Bart, 2005, « Postclassical Narratology », dans D. Herman, M. Jahn et M. L. Ryan (dir.), The Routledge Encyclopedia of Narrative Theory, Londres, Routledge, p. 450-451.

Kendall, Paul Murray, 1965, The Art of Biography, Londres, George Allen \& Unwin.

Lavocat, Françoise, 2016, Fait et fiction : pour une frontière, Paris, Seuil.

Lecarme, Jacques, 2004, "Origines et évolution de la notion d'autofiction », dans B. Blanckeman, A. Mura-Brunel et M. Dambre (dir.), Le Roman français au tournant du XXI siècle, Paris, Presses Sorbonne Nouvelle, p. 13-23.

Lejeune, Philippe, 1975, Le Pacte autobiographique, Paris, Seuil.

Lejeune, Philippe, 1986, Moi aussi, Paris, Seuil.

Löshnigg, Martin, 2010, « Postclassical Narratology and Autobiography », dans J. Alber et M. Fludernik (dir.), Postclassical Narratology: Approaches and Analyses, Columbus, Ohio State University Press, p. 255-274.

Madelénat, Daniel, 2007, « Le vrai, le faux, le figuré : à propos de David Bellos, Georges Perec : Une vie dans les mots (Seuil, 1994) et d'Antibiotiques (Cahiers Georges Perec $\mathrm{n}^{\circ}$ 7, Le Castor Astral, 2003) ", dans A.-M. Monluçon et A. Salha (dir.), Fictions biographiques XIX ${ }^{e}-X^{e}$ siècles, avec la participation scientifique de Brigitte Ferrato-Combe, Toulouse, Presses universitaires du Mirail, p. 289-304.

Madelénat, Daniel, 2013, « L'auteur ! L'auteur ! Biographie, l'as-tu vu ? », dans Robert Dion et Frédéric Regard (dir.), Les Nouvelles Écritures biographiques, Lyon, ENS éditions, p. 59-72.

Martin, Wallace, 1986, Recent Theories of Narrative, Ithaca, New York, Londres, Cornell University Press.

Martinez, Matías et Scheffel, Michael, 2003, « Narratology and Theory of Fiction. Remarks on a Complex Relationship », dans T. Kindt et H.H. Müller (dir.), What is Narratology? Questions and Answers Regarding the Status of a Theory, Berlin, Walter de Gruyter.

Michon, Pierre, 1984, Vies minuscules, Paris, Gallimard.

Monluçon, Anne-Marie et Salha, Agathe (dir.), 2007, Fictions biographiques XIX ${ }^{e}-X X^{e}$ siècles, avec la participation scientifique de Brigitte Ferrato-Combe, Toulouse, Presses Universitaires du Mirail.

Nünning, Ansgar, 2005, « How to Distinguish Between Fictional and Factual Narratives: Narratological and Systemtheoritical Suggestions », dans L. Å. Skalin (dir.), Fact and Fiction in Narrative: An Interdisciplinary Approach, Örebro, Univ. Library, p. 23-56. 
Pavel, Thomas, [1986] 1988, Univers de la fiction, trad. de l'anglais et remanié par l'auteur, Paris, Seuil.

Pavel, Thomas, 2001, « Comment définir la fiction? », dans R. Audet et A. Gefen (dir.), Frontières de la fiction, Québec, Nota Bene, Bordeaux, Presses universitaires de Bordeaux, p. 3-13.

Richard, Jean-Pierre, 1990, L'État des choses. Études sur huit écrivains d'aujourd'hui, Paris, Gallimard. Robin, Régine, 1998, Le Golem de l'écriture. De l'autofiction au cybersoi, Montréal, XYZ.

Ryan, Marie-Laure, 2001, « Frontière de la fiction : digitale ou analogique ? », dans A. Gefen et R. Audet (dir.), Frontières de la fiction, Québec, Nota Bene, Bordeaux, Presses Universitaires de Bordeaux, p. 17-42.

Schaeffer, Jean-Marie, 1989, Qu'est-ce qu'un genre littéraire?, Paris, Seuil.

Schaeffer, Jean-Marie, 1999, Pourquoi la fiction ?, Paris, Seuil.

Schwob, Marcel, 1896, « Préface » aux Vies imaginaires, Paris, Bibliothèque-Charpentier, p. 1-21.

Searle, John R., [1975] 1982, "Le statut logique du discours de fiction » [ "The Logical Status of Fictional Discourse »], dans Sens et expression. Études de théorie des actes de langage, trad. et préface de Joëlle Proust, Paris, Minuit, p. 101-119.

Skalin, Lars-Åke (dir.), 2005, Fact and Fiction in Narrative: An Interdisciplinary Approach, Örebro, Univ. Library.

Skalin, Lars-Åke (dir.), 2008, Narrativity, Fictionality and Literariness. The Narrative Turn and the Study of Literary Fiction, Örebro, Örebro Studies in Literary History and Criticism, $\mathrm{n}^{\circ} 7$.

Viart, Dominique, 2002, « Dis moi qui te hante », Revue des Sciences humaines, « Paradoxes du biographique $», n^{\circ} 263$, p. 7-33.

Viart, Dominique et Vercier, Bruno, 2005, La Littérature française au présent. Héritage, modernité, mutations, Paris, Bordas.

White, Hayden, 1973, Metahistory. The Historical Imagination in Nineteenth Century Europe, Baltimore, The Johns Hopkins University Press.

White, Hayden, 1987, The Content of Form: Narrative Discourse and Historical Representation, Baltimore, John Hopkins University Press.

White, Hayden, 1999, Figural Realism: Studies in the Mimesis Effect, Baltimore, John Hopkins University Press.

\section{NOTES}

1. Tandis que le conditionnalisme, en fondant la fictionnalité sur une critique du jugement, postule l'existence de critères distinctifs de la fiction, mais fait inévitablement varier ces derniers en fonction des usages qu'un lecteur choisit de faire d'un texte donné (ce qui correspond à la démarche de Lejeune), l'essentialisme défend l'hypothèse selon laquelle c'est à l'intérieur des textes eux-mêmes qu'il conviendrait de chercher les signes distinctifs du discours fictionnel (comme c'est le cas de Dorrit Cohn).

2. Isabelle Grell, «À propos de la chose commune : l'autofiction », entretien de Konan Kouassi Samuel avec Isabelle Grell, date de parution inconnue, http://www.autofiction.org/index.php? post/2015/09/10/A-propos-de-la-chose-commune-\%3A-lAutofiction, consulté le 4 octobre 2016. 
3. Tandis que les théories de Käte Hamburger postulent l'existence de marqueurs internes de fictionnalité, John Searle et Gérard Genette ont fait reposer la fictionnalité sur des critères externes au texte, tels que l'intention illocutoire de l'auteur. J'y reviendrai.

4. Ma traduction: "Postclassical narratology (which should not be conflated with poststructuralist theories of narrative) contains classical narratology as one of its "moments" but is marked by a profusion of new methodologies and research hypothesis: the result is a host of new perspectives on the forms and functions of narrative itself. »

5. Ma traduction : "Whereas structuralism was intent on coming up with a general theory of narrative, postclassical narratology prefers to consider the circumstances that make every act of reading different. "

6. Voir par exemple Schaeffer (1989: 80-82) : « une œuvre littéraire, comme tout acte discursif, est une réalité sémiotique complexe et pluridimensionnelle; de ce fait, la question de son identité ne saurait avoir de réponse unique [...]. Pour qu'il y ait un acte discursif, il faut que le support communicationnel soit investi d'une intention de communication [...]. Le support matériel ne devient une réalité sémantique et syntaxique que parce qu'il est investi par un acte communicationnel [...]: il est un événement, mais un événement qui exprime une intentionnalité. »

7. Les termes « analogique » et « digital » renvoient métaphoriquement à une distinction opérée par le physicien Niels Bohr, qui a montré qu'il « est parfois plus avantageux de considérer la lumière comme une onde (l'analogique), parfois plus efficace de la décrire comme formée de particules (le digital)» (Ryan 2001 : 37). Pour plus de clarté, je préfère généralement qualifier le modèle "digital » théorisé par Marie-Laure Ryan de "binaire », car j'estime cette appellation plus explicite.

8. «Paratactique, adj. (Propositions) paratactiques. (Propositions) juxtaposées sans outil de coordination ou de subordination. (Ds Mar. Lex. 1951). LING. Juxtaposition de deux propositions entre lesquelles le lien de dépendance n'est qu'implicite, la courbe mélodique commune dispensant de l'usage d'un outil de coordination ou de subordination » (Source : Centre national de ressources textuelles et lexicales: http://www.cnrtl.fr/definition/paratactique, consulté le 8 octobre 2016).

9. Ma traduction: "The Zionist historical interpretation of the Holocaust, White says, is not a contre-vérité (as has been suggested by Vidal-Naquet) but a truth: "its truth, as a historical interpretation, consists precisely in its effectiveness (my italics) in justifying a wide range of current Israeli political policies that, from the standpoint of those who articulate them, are crucial to the security and indeed the very existence of Jewish people." In the same way, "the effort of the Palestinian people to mount a politically effective (my italics) response to the Israeli policies entails the production of a similarly effective (my italics) ideology, complete with an interpretation of their history capable of endowing it with a meaning that it has hitherto lacked." We can conclude that if Faurisson's narrative were ever to prove effective, it would be regarded by White as true as well. »

10. Françoise Lavocat (2016: 35) cite, à titre d'exemple: Banfield (1995 [1982], Martin (1986), Cohn ([1999] 2001), Martinez et Scheffel (2003), Nünning (2005), Skalin (dir.) (2005).

11. Agnès Blesch et Pauline Mulin, "L'un et l'autre", disponible sur le site Écritures contemporaines: atelier de recherche sur la littérature actuelle, http://ecrit-cont.ens-lyon.fr/ spip.php?rubrique62 (consulté le 2 mars 2015). Le présent article vise entre autres à remettre en question une telle caractérisation générique du genre biofictif.

12. Il y a quelques années, Daniel Madelénat (2013: 69-70) soulignait de cette manière la difficulté de théoriser le genre biofictif : «Énumérer des noms - Pierre Michon, Pascal Quignard, Patrick Modiano, Claude Louis-Combet, Jean-Benoît Puech, Gérard Macé, Richard Millet... - et des traits (déflation, parfois jusqu'au laconisme; transgression multipliée des limites génériques; « contrevie », comme auto-, hétéro-, biofiction ; autocommentaire et métadiscours du biographe ; 
irruption du conditionnel, de l'incertitude ou de la contrefactualité... demanderait tout un essai (hasardeux s'il prétendait rationaliser la fluidité d'un mouvement diffus).»

13. Le premier ouvrage de Serge Gasparini traitant de ce sujet propose un survol très succinct de ces débats (2004:22-26).

14. Philippe Gasparini (2008: 116) commente le retour de Genette à une conception spécifiquement fictionnelle du genre autofictif à partir de l'analyse qu'en a fait Vincent Colonna - dont Gérard Genette a d'ailleurs dirigé la thèse de doctorat: "Je parle ici des vraies autofictions - dont le contenu narratif est, si j'ose dire, authentiquement fictionnel, comme (je suppose) celui de La Divine Comédie - et non des fausses autofictions, qui ne sont “-fictions" que pour la douane : autrement dit, autobiographies honteuses» (Genette 1991 : 86-87).

\section{RÉSUMÉS}

Cet article explore un problème théorique lié aux considérations génériques que soulèvent l'autobiographie, l'autofiction, la biographie et la biofiction, dans le contexte plus général des débats récents portant sur la distinction logique et ontologique entre fait et fiction. Il cherche à démontrer que les différentes typologies visant à distinguer les récits de vie des trente à quarante dernières années en fonction de leur degré de fictionnalité ou de référentialité adoptent pour la majorité une posture que Gérard Genette a qualifiée de "conditionnaliste ", puisqu'elle fonde la fictionnalité sur une critique du jugement, par opposition aux théories dites « essentialistes » qui postulent l'existence de marqueurs internes de la fiction.

Plus spécifiquement, mon article s'intéresse à l'impact que cette approche méthodologique a produit sur la théorie des genres, en proposant que celle-ci fait aujourd'hui l'objet d'une polarisation entre deux tendances. Tandis que la première a abouti à la multiplication de catégories génériques visant à apposer un caractère normatif au "pacte de lecture » qu'instaurent des œuvres singulières, la seconde consiste à l'inverse en l'effacement des frontières génériques au profit d'une analyse thématique de la littérature contemporaine.

This article investigates a theoretical problem linked to generic speculations about autobiography, autofiction, biography and biofiction, in the broader context of recent debates concerning the logical and ontological distinction between fact and fiction. It aims to demonstrate that the different typologies which seek to classify contemporary life tales according to their degree of fictionality or referentiality adopt for the most part a methodological position described by Gerard Genette as "conditionnalist," for it considers that fictionality results from an act of judgment, as opposed to the "essentialist" posture which postulates the existence of internal markers of fictionality.

More specifically, my article seeks to evaluate the impact that the conditionnalist posture may have produced on the theory of genres, arguing that the latter is now polarized between two tendencies. Whereas the prescriptive/normative attitude incites critics to multiply generic labels in an effort to systematize author-reader contracts, which are always unique, the descriptive/ typological attitude tends, on the contrary, to dissolve generic borders in favor of a thematic analysis of contemporary literature. 
INDEX

Keywords : fictionality, referentiality, genre theory, postclassical narratology, autobiography, biofiction

Mots-clés : fictionnalité, référentialité, théorie des genres littéraires, narratologie postclassique, autobiographie, biofiction

\section{AUTEUR}

\section{MIRUNA CRACIUNESCU}

Université McGill (Montréal), Affiliée à la Chaire de recherche James McGill en études de la Renaissance 